This document is the accepted manuscript version of the following article:

Lu, T., Huang, J., Jordi, C., Kovacs, G., Huang, R., Clarke, D. R., \& Suo, Z.

(2012). Dielectric elastomer actuators under equal-biaxial forces, uniaxial forces, and uniaxial constraint of stiff fibers. Soft Matter, 8(22), 6167-6173.

https://doi.org/10.1039/C2SM25692D

\title{
Dielectric elastomer actuators under equal-biaxial forces, uniaxial forces, and uniaxial constraint of stiff fibers
}

Tongqing Lü ${ }^{1,2}$, Jiangshui Huang², Christa Jordi³, Gabor Kovacs33, Rui Huang4, David R. Clarke² and Zhigang Suo ${ }^{2, a}$

${ }^{1}$ School of Aerospace, Xi'an Jiaotong University, Xi'an, 710049, P.R. China

${ }^{2}$ School of Engineering and Applied Sciences, Harvard University, Cambridge, MA 02138, USA

3 Empa, Swiss Federal Laboratories for Material Science and Technology, Überlandstrasse 129, 8600 Dübendorf, Switzerland

4 Department of Aerospace Engineering and Engineering Mechanics, University of Texas, Austin, TX 78712, USA

\begin{abstract}
A membrane of a dielectric elastomer deforms when a voltage is applied through its thickness. The achievable voltage-induced deformation is strongly affected by how mechanical loads are applied. Large voltage-induced deformation has been demonstrated for a membrane under equal-biaxial forces, but only small voltage-induced deformation has been observed for a membrane under a uniaxial force. This difference is interpreted here theoretically. The theory also predicts that, when the deformation of a membrane is constrained in one direction, a voltage applied through the thickness of the membrane can cause it to deform substantially in the other direction. Experiments are performed on membranes under equal-biaxial forces and uniaxial forces, as well as on fiber-constrained membranes of two types: a dielectric elastomer membrane with carbon fibers on both faces, and two dielectric elastomer membranes sandwiching nylon fibers. The experimental observations are compared with the theory.
\end{abstract}

keywords: dielectric elastomer, fiber-constrained, unidirectional actuation, soft robot, energy harvesting.

a Corresponding author, email: suo@seas.harvard.edu 


\section{Introduction}

A dielectric elastomer deforms in response to an applied voltage. This phenomenon is being studied intensely in developing dielectric elastomer transducers, a technology also known as artificial muscles. ${ }^{1,2}$ Attributes of dielectric elastomer transducers include large deformation, fast response, light weight, silent operation and low cost. ${ }^{3,4}$ The technology is promising for broad applications, such as actuators for soft robots and MEMS, ${ }^{5-8}$ tunable lenses and haptic interfaces for mobile phones, ${ }^{9,10}$ and generators for harvesting energy from human movements and ocean waves..$^{11,12}$

A membrane of a dielectric elastomer in a transducer is sandwiched between two compliant electrodes (Fig. 1). When the electrodes are subject to a voltage, the positive and negative charges spread on the two faces of the membrane, so that the membrane reduces in thickness and expands in area. The achievable voltage-induced deformation is strongly affected by how mechanical loads are applied. Using an acrylic elastomer, experiments have demonstrated voltage-induced expansion in area by $158 \%$ with a membrane biaxially prestretched and fixed to a rigid frame, ${ }^{1}$ by $488 \%$ with a membrane subject to biaxial dead loads, ${ }^{13}$ and by $1689 \%$ with a membrane mounted on a chamber of air. ${ }^{14}$

Despite these achievements, much of the operational space of dielectric elastomer transducers remains uncharted. The effect of prestretches has been attributed to eliminating the pull-in instability, ${ }^{15}$ or improving electric breakdown strength, ${ }^{16,17}$ or actuating on the verge of the pull-in instability without electric breakdown. ${ }^{14}$ However, direct, quantitative comparison between theory and experiment is lacking. Furthermore, many applications require muscle-like actuators in which voltage induces large and unidirectional displacement. While large voltage-induced deformation has been demonstrated for a membrane under equal-biaxial forces, only small voltage-induced deformation has been observed for a membrane under a uniaxial force. This difference has so far remained unexplained.

Here we make a theoretical comparison of electromechanical responses of membranes under several conditions. For a membrane under equal-biaxial forces, the voltage-induced strain reaches a maximum at forces of an intermediate magnitude, when the type of failure changes from electromechanical instability to electric breakdown. For a 
membrane under a uniaxial-force, the voltage-induced strain in the direction of applied force reduces as the force increases. For a membrane with stiffeners that restrict deformation in one direction, large voltage-induced deformation in the other direction is achievable.

We demonstrate large, unidirectional, voltage-induced displacement by constraining the membrane in the other direction using stiff fibers. Our work has been motivated by several experimental observations. For a membrane pulled and fixed to a certain length in one direction, upon subjecting to a voltage through the thickness, the membrane can expand several times in the other direction. ${ }^{1}$ In another setup, a membrane is pulled horizontally, fixed by two rigid clamps on the upper and lower edges, and subjected to a mechanical force in the vertical direction; voltage-induced linear strain up to $360 \%$ have been achieved. ${ }^{18}$ To constrain the horizontal prestretch, this setup using clamps requires a membrane long in the horizontal direction and short in the vertical direction. Membranes of such a shape are unsuitable for applications that require long artificial muscles to give large voltage-induced displacement. Yet another setup involves sandwiching a membrane between corrugated metallic electrodes; voltage-induced linear strains of a few percent have been reported. ${ }^{19}$ It has been mentioned that unidirectional actuation may be achieved by constraining deformation in the other direction using stiffeners. ${ }^{20}$ Several theoretical papers have studied voltage-induced deformation in anisotropic membranes. ${ }^{21-25}$

We perform experiments under three conditions. In experiments under equal biaxial conditions, voltage-induced linear strains up to $155 \%$ are observed, and the change of the type of failure is captured. In experiments with membranes under uniaxial forces, voltage-induced linear strains up to $22 \%$ are observed, and the predicted decrease of the maximum actuation with increasing applied force is verified. We also make two types of fiber-constrained dielectric elastomer membranes: a membrane with carbon fibers on both faces, and two membranes sandwiching nylon fibers. Voltage-induced unidirectional strains up to $28 \%$ are observed.

\section{Voltage-induced strains for membranes under several conditions}

This section uses the theory of dielectric elastomers to compare electromechanical responses of membranes under several conditions: a membrane under equal-biaxial forces, 
a membrane under a uniaxial force, and a membrane constrained in one direction and subject to a force in the other direction. Membranes under each of the three conditions can exhibit complex behavior, and have been studied separately.15,18,21,26 Here we compare aspects of direct relevance to this work. Specifically, the theory accounts for the observations that large voltage-induced deformation is achievable for a membrane under equal-biaxial forces, but not for a membrane under a uniaxial force. Furthermore, the theory predicts that fiber-constrained membranes can achieve large, unidirectional, voltage-induced displacement.

\subsection{Theory of dielectric elastomers}

The theory of dielectric elastomers has been reviewed recently. ${ }^{27}$ Here we summarize the key ideas relevant to this work. A membrane of a dielectric elastomer deforms in response to applied forces and voltage (Fig. 1). In the reference state, subject to no force and no voltage, the membrane is of thickness $H$ and lengths $L_{1}$ and $L_{2}$. In a deformed state, subject to forces $P_{1}$ and $P_{2}$ in the plane and voltage $\Phi$ through the thickness, the membrane is of thickness $h$ and lengths $l_{1}$ and $l_{2}$. Define the stresses as $\sigma_{1}=P_{1} /\left(l_{2} h\right)$ and $\sigma_{2}=P_{2} /\left(l_{1} h\right)$, the electric field as $E=\Phi / h$, and the stretches as $\lambda_{1}=l_{1} / L_{1}$ and $\lambda_{2}=l_{2} / L_{2}$.

We adopt the model of ideal dielectric elastomers. This model assumes that the membrane is incompressible $\left(h l_{1} l_{2}=H L_{1} L_{2}\right)$, and that the permittivity $\varepsilon$ is a constant independent of deformation. The equations of state for an ideal dielectric elastomer are

$$
\begin{gathered}
\sigma_{1}+\varepsilon E^{2}=\lambda_{1} \frac{\partial W_{\text {stretch }}\left(\lambda_{1}, \lambda_{2}\right)}{\partial \lambda_{1}}, \\
\sigma_{2}+\varepsilon E^{2}=\lambda_{2} \frac{\partial W_{\text {stretch }}\left(\lambda_{1}, \lambda_{2}\right)}{\partial \lambda_{2}},
\end{gathered}
$$

where $W_{\text {stretch }}\left(\lambda_{1}, \lambda_{2}\right)$ is the Helmholtz free energy associated with stretching the elastomer. The elasticity of the elastomer is balanced by the applied stresses $\sigma_{1}$ and $\sigma_{2}$, along with 
the electrostatic interaction-the Maxwell stress $\varepsilon E^{2}$.

We adopt a free energy function that captures the stretch-stiffening behavior. In an elastomer, each individual polymer chain has a finite contour length. When the elastomer is subject to no loads, the polymer chains are coiled, allowing a large number of conformations. Subject to loads, the polymer chains become less coiled. As the loads increase, the end-to-end distance of each polymer chain approaches the finite contour length, and the elastomer approaches a limiting stretch. On approaching the limiting stretch, the elastomer stiffens steeply. To account for this behavior, we adopt the Gent model: ${ }^{28}$

$$
W_{\text {stretch }}\left(\lambda_{1}, \lambda_{2}\right)=-\frac{\mu}{2} J_{\lim } \log \left(1-\frac{\lambda_{1}^{2}+\lambda_{2}^{2}+\lambda_{1}^{-2} \lambda_{2}^{-2}-3}{J_{\lim }}\right),
$$

where $\mu$ is the shear modulus, and $J_{\lim }$ is a material constant related to the limiting stretch. When the stretches are small, $\lambda_{1}^{2}+\lambda_{2}^{2}+\lambda_{1}^{-2} \lambda_{2}^{-2}-3<<J_{\lim }$, the Gent model recovers the neo-Hookean model, $W_{\text {stretch }}\left(\lambda_{1}, \lambda_{2}\right)=(\mu / 2)\left(\lambda_{1}^{2}+\lambda_{2}^{2}+\lambda_{1}^{-2} \lambda_{2}^{-2}-3\right)$. When the stretches approach the limit, $\lambda_{1}^{2}+\lambda_{2}^{2}+\lambda_{1}^{-2} \lambda_{2}^{-2}-3 \rightarrow J_{\lim }$, the Gent model stiffens steeply.

A combination of (1)-(3) gives the equations of state:

$$
\begin{aligned}
\sigma_{1}+\varepsilon E^{2} & =\frac{\mu\left(\lambda_{1}^{2}-\lambda_{1}^{-2} \lambda_{2}^{-2}\right)}{1-\left(\lambda_{1}^{2}+\lambda_{2}^{2}+\lambda_{1}^{-2} \lambda_{2}^{-2}-3\right) / J_{\lim }}, \\
\sigma_{2}+\varepsilon E^{2} & =\frac{\mu\left(\lambda_{2}^{2}-\lambda_{1}^{-2} \lambda_{2}^{-2}\right)}{1-\left(\lambda_{1}^{2}+\lambda_{2}^{2}+\lambda_{1}^{-2} \lambda_{2}^{-2}-3\right) / J_{\lim }} .
\end{aligned}
$$

Throughout the paper, we consider an acrylic elastomer VHB (by $3 \mathrm{M}$ ) with the following representative material parameters: $\varepsilon=3.98 \times 10^{-11} \mathrm{~F} / \mathrm{m}$, and $J_{\lim }=120.13,17$ VHB exhibits appreciable viscoelasticity; the shear modulus $\mu$ is varied from $45 \mathrm{kPa}$ to $65 \mathrm{kPa}$ to fit experimental data. The thickness of a membrane in the undeformed state is $H=1 \mathrm{~mm}$ for VHB4910 and $H=0.5 \mathrm{~mm}$ for VHB4905. We also adopt a constant value of electric breakdown field $E_{E B}=250 \mathrm{MV} / \mathrm{m}^{16,18,29,30}$

\subsection{Actuation of a membrane under equal-biaxial conditions}

Consider the actuation of a membrane under the equal-biaxial conditions (Fig. 2a). 
In the reference state, a square membrane, length $L$ and thickness $H$, is subject to no force and no voltage. In the prestretched state, subject to equal-biaxial force $P$, the membrane is stretched to length $\lambda^{\text {pre }} L$. In the actuated state, subject to both the force $P$ and the voltage $\Phi$, the membrane has stretches $\lambda_{1}=\lambda_{2}=\lambda$, the stresses $\sigma_{1}=\sigma_{2}=\lambda P /(L H)$ and the electric field $E=\lambda^{2} \Phi / H$. Equations of state (4) and (5) reduce to

$$
\lambda \frac{P}{L H}+\varepsilon\left(\lambda^{2} \frac{\Phi}{H}\right)^{2}=\frac{\mu\left(\lambda^{2}-\lambda^{-4}\right)}{1-\left(2 \lambda^{2}+\lambda^{-4}-3\right) / J_{\lim }}
$$

This equation determines the stretch $\lambda$ of a membrane subject to given values of $P$ and $\Phi$.

For a fixed force $P$, as the voltage $\Phi$ varies, the membrane deforms in a succession of states of equilibrium, represented by a curve in the $\Phi-\lambda$ diagram (Fig. 2b). A fixed force $P$ sets a value of the prestretch $\lambda^{\text {pre }}$, which for each curve is read from the intersection of the curve and the horizontal axis. For given values of $\lambda^{\text {pre }}$ and $\Phi$, the membrane can be in one or multiple states of equilibrium. ${ }^{15}$ When the prestretch $\lambda^{\text {pre }}$ is small, the $\Phi-\lambda$ curve is N-shaped-going up, down, and then up again. When the prestretch $\lambda^{\text {pre }}$ is large, the $\Phi-\lambda$ curve increases monotonically.

The two types of $\Phi-\lambda$ curves give qualitatively different electromechanical responses. ${ }^{31}$ When the $\Phi-\lambda$ curve is N-shaped, the membrane is prone to pull-in instability. In an experiment in which the voltage is programmed to ramp up slowly, when the voltage reaches the local maximum, no state of equilibrium exists nearby as the voltage goes up further; rather, the membrane snaps to a state of equilibrium with a much larger stretch. Corresponding to the larger stretch, the membrane becomes much thinner, and the electric field in the membrane becomes much higher. For the parameters used in this paper, the $\Phi-\lambda$ curve changes from being $\mathrm{N}$-shaped to being monotonic at a prestretch of $\lambda^{\text {pre }} \approx 2.21$.

Also plotted in the voltage-stretch diagram is the curve representing the electric-breakdown conditions, $E_{E B}=\lambda^{2} \Phi / H$ (Fig. 2b). When $\lambda^{\text {pre }}<1.75$, the $\Phi-\lambda$ curve is $\mathrm{N}$-shaped, electric breakdown occurs before the membrane reaches the state of 
equilibrium of larger stretch, and the maximum stretch $\lambda^{\max }$ usable for actuation is limited by the stretch when the voltage reaches the peak. When the $1.75<\lambda^{\text {pre }}<2.21$, the $\Phi-\lambda$ curve is still $\mathrm{N}$-shaped, but the membrane snaps to the state of equilibrium of larger stretch without electric breakdown, so that the maximum stretch $\lambda^{\max }$ usable for actuation is limited by electric breakdown. When $\lambda^{\text {pre }}>2.21$, the $\Phi-\lambda$ curve is monotonic, and the maximum stretch $\lambda^{\max }$ usable for actuation is also limited by electric breakdown.

The maximum actuation, defined as $\lambda^{\max } / \lambda^{\text {pre }}$, is a function of the prestretch $\lambda^{\text {pre }}$ (Fig. 2c). When $\lambda^{\text {pre }}<1.75$, the maximum actuation is limited by the pull-in instability, and increases somewhat as the prestretch increases. In this regime, because the pull-in instability is entirely determined by the equations of state, the maximum actuation is independent of the electric breakdown strength. By contrast, when $\lambda^{\text {pre }}>1.75$, the maximum actuation is limited by electric breakdown, and decreases as the prestretch increases. In this regime, because $\lambda^{\max }$ is given by the intersection between the voltage-stretch curve and the condition of electric breakdown, which appears to be insensitive to the prestretch, the maximum actuation $\lambda^{\max } / \lambda^{\text {pre }}$ is nearly inversely proportional to $\lambda^{\text {pre }}$. At $\lambda^{\text {pre }}=1.75$, the mode of failure switches from the pull-in instability to electric breakdown, and the maximum actuation $\lambda^{\max } / \lambda^{\text {pre }}$ is large. This transition of the mode of failure accounts for the experimentally observed prestretch-enhanced actuation. ${ }^{13,15}$

\subsection{Actuation of a membrane under a uniaxial force}

Next consider the actuation of a membrane under a uniaxial force (Fig. 3a). In the reference state, subject to no force and no voltage, the membrane is of thickness $H$ and length $L_{1}$ and $L_{2}$. In the prestretched state, subject to the force $P$ in the vertical direction, the membrane elongates to $\lambda_{2}^{\text {pre }} L_{2}$. In an actuated state, subject to the force $P$ in the vertical direction and voltage $\Phi$ through the thickness, the membrane has stretches $\lambda_{1}$ 
and $\lambda_{2}$, stresses $\sigma_{1}=0$ and $\sigma_{2}=\lambda_{2} P /\left(L_{1} H\right)$, and electric field $E=\lambda_{1} \lambda_{2} \Phi / H$. Equations of state (4) and (5) reduce to

$$
\begin{gathered}
\varepsilon\left(\lambda_{1} \lambda_{2} \frac{\Phi}{H}\right)^{2}=\frac{\mu\left(\lambda_{1}^{2}-\lambda_{1}^{-2} \lambda_{2}^{-2}\right)}{1-\left(\lambda_{1}^{2}+\lambda_{2}^{2}+\lambda_{1}^{-2} \lambda_{2}^{-2}-3\right) / J_{\lim }}, \\
\lambda_{2} \frac{P}{L_{1} H}+\varepsilon\left(\lambda_{1} \lambda_{2} \frac{\Phi}{H}\right)^{2}=\frac{\mu\left(\lambda_{2}^{2}-\lambda_{1}^{-2} \lambda_{2}^{-2}\right)}{1-\left(\lambda_{1}^{2}+\lambda_{2}^{2}+\lambda_{1}^{-2} \lambda_{2}^{-2}-3\right) / J_{\lim }} .
\end{gathered}
$$

The two equations determine the two stretches $\lambda_{1}$ and $\lambda_{2}$ of a membrane subject to given values of $P$ and $\Phi$.

For a given $P$, as the voltage $\Phi$ varies, the membrane deforms in a succession of states of equilibrium, represented by a curve in the $\Phi-\lambda_{2}$ diagram (Fig. 3b). The value of the prestretch $\lambda_{2}^{\text {pre }}$ for each curve is read from the intersection of the curve and the horizontal axis. For every value of $P$ plotted here, the $\Phi-\lambda_{2}$ curve is N-shaped. Also plotted in the voltage-stretch diagram is the curve representing the electric-breakdown conditions, $E_{E B}=\lambda_{1} \lambda_{2} \Phi / H$. For the parameters used here, the actuation of a membrane under a uniaxial force is limited by electromechanical instability. A detailed discussion of a membrane under a uniaxial force is available elasewhere. ${ }^{26}$

The maximum actuation, defined as $\lambda_{2}^{\max } / \lambda_{2}^{\text {pre }}$, is a function of the prestretch $\lambda_{2}^{\text {pre }}$ (Fig. 3c). The larger the vertical force is applied, the smaller the actuation is achievable. This behavior is understood as follows. When the force is applied in the vertical direction, the membrane stiffens in the vertical direction, but remains compliant in the horizontal direction. This compliance allows the membrane under the voltage to deform readily in the horizontal direction and thin down drastically, leading to the $\mathrm{N}$-shaped voltage-stretch curves. Rather than enhancing the achievable actuation strain, a uniaxial force reduces it. This theoretical prediction accounts for small actuation strains for membranes under uniaxial forces.

\subsection{Actuation of a fiber-constrained membrane}


By contrast, large actuation in the vertical direction has been observed when the horizontal deformation is constrained.,18 Here we constrain horizontal deformation by using stiff fibers (Fig. 4). In the reference state, the membrane is subject to no force and no voltage. The membrane is then stretched in both directions and fibers are added to align in the horizontal direction. The prestretch in the horizontal direction $\left(\lambda_{1}^{\text {pre }}\right)$ can be tuned over a wide range by adjusting the mechanical forces. When the mechanical forces are released, the horizontal length of the membrane is fixed by the fibers at $\lambda_{1}^{\text {pre }} L_{1}$, and the vertical length of the membrane contracts to $\left(\lambda_{1}^{\text {pre }}\right)^{-1 / 2} L_{2}$. Next, the membrane is subject to a force $P$ in the vertical direction so that the vertical prestretch becomes $\lambda_{2}^{\text {pre }}$, while the horizontal prestretch remains to be $\lambda_{1}^{\text {pre }}$. In the actuated state, the membrane is subject to both the force $P$ and the voltage $\Phi$, the horizontal stretch is assumed to be fixed by the fibers $\lambda_{1}=\lambda_{1}^{\text {pre }}$, but the vertical stretch becomes $\lambda_{2}$. Equations of state (4) and (5) become

$$
\begin{gathered}
\lambda_{2} \frac{P}{L_{1} H}+\varepsilon\left(\lambda_{1}^{\text {pre }} \lambda_{2} \frac{\Phi}{H}\right)^{2}=\frac{\mu\left(\lambda_{2}^{2}-\left(\lambda_{1}^{\text {pre }} \lambda_{2}\right)^{-2}\right)}{1-\left(\left(\lambda_{1}^{\text {pre }}\right)^{2}+\lambda_{2}^{2}+\left(\lambda_{1}^{\text {pre }} \lambda_{2}\right)^{-2}-3\right) / J_{\lim }}, \\
\sigma_{1}+\varepsilon\left(\lambda_{1}^{\text {pre }} \lambda_{2} \frac{\Phi}{H}\right)^{2}=\frac{\mu\left(\left(\lambda_{1}^{\text {pre }}\right)^{2}-\left(\lambda_{1}^{\text {pre }} \lambda_{2}\right)^{-2}\right)}{1-\left(\left(\lambda_{1}^{\text {pre }}\right)^{2}+\lambda_{2}^{2}+\left(\lambda_{1}^{\text {pre }} \lambda_{2}\right)^{-2}-3\right) / J_{\lim }} .
\end{gathered}
$$

Once actuated with given values of $P$ and $\Phi$, the vertical stretch $\lambda_{2}$ is determined from (9), and the horizontal stress $\sigma_{1}$ is determined from (10).

For a given $P$, as the voltage $\Phi$ varies, the membrane deforms in a succession of states of equilibrium, represented by a curve in the $\Phi-\lambda_{2}$ diagram (Fig. 5). The value of $\lambda_{2}^{\text {pre }}$ for each curve is read from the intersection of the curve and the horizontal axis. All the $\Phi-\lambda_{2}$ curves with fixed values of the horizontal stretch are monotonic. Also plotted in the voltage-stretch diagram is the curve representing the electric-breakdown conditions, $E_{E B}=\lambda_{1} \lambda_{2} \Phi / H$. 
The horizontal stretch of the membrane may not be fully constrained by the fibers. When the horizontal stress in the membrane is tensile, the fibers are under compression and may buckle. The buckled fibers may partially release the tensile stress in the membrane. When the fibers are under tension, the horizontal stress in the membrane is compressive. It is commonly assumed that membrane cannot sustain compressive stress, and will form wrinkles. ${ }^{18}$ It remains unclear how densely packed fibers will effectively constrain the horizontal stretch of the membrane. The equations of state (9) and (10) assume that the horizontal stretch is perfectly constrained, without fiber buckling or membrane wrinkling. In the voltage-stretch diagrams (Fig. 5) we mark the condition of loss of tension $\left(\sigma_{1}=0\right)$, beyond which the horizontal stretch of the membrane is no longer perfectly constrained. Although the loss of tension may not cause immediate failure, ${ }^{18}$ here we use the loss of tension to define allowable actuation, $\lambda_{2}^{\max } / \lambda_{2}^{\text {pre }}$, which may be considered as a lower bound for maximum actuation. The maximum actuation $\lambda_{2}^{\max } / \lambda_{2}^{\text {pre }}$ is a function of the prestretches $\lambda_{1}^{\text {pre }}$ and $\lambda_{2}^{\text {pre }}$ (Fig. 6). Large, unidirectional, voltage-induced strains are predicted for the cases with $\lambda_{1}^{\text {pre }}>1$.

\section{Experiments on dielectric elastomer actuators under different conditions}

\subsection{Actuation of membranes under equal-biaxial forces and uniaxial forces}

To ascertain the theoretical predictions, we perform experiments following the procedures described elsewhere using VHB membranes under equal-biaxial forces ${ }^{13}$ and uniaxial forces. $3^{2}$ For membranes under equal-biaxial forces, the experiment captures the transition from failure limited by electromechanical instability to that limited by electric breakdown (Fig. 2). For membranes under uniaxial forces, the experiment confirms the theoretical prediction that the maximum actuation decreases as the applied force increases (Fig, 3). In both cases, the experimental data and the theoretical predictions agree remarkably well. Two values of the shear modulus, $\mu=45 \mathrm{kPa}$ and $\mu=65 \mathrm{kPa}$, are used to fit the two sets of experimental data. The variation in the shear modulus mainly results from the viscous effect of VHB. 
3.2 Actuator made by placing carbon fibers on both surfaces of a membrane

A membrane of VHB $4910(3 \mathrm{M})$ is pulled to a horizontal stretch of 3 and a vertical stretch of 2.5, and is fixed on a rigid frame. A mask made of a large wax paper with a rectangular hole of size $100 \mathrm{mmx} 40 \mathrm{~mm}$ was stuck to the stretched VHB membrane with width in horizontal direction and length in vertical direction, and then the carbon fibers (192 UD STS 5631 by Texero) are applied horizontally. The active area measures $84 x 40 \mathrm{~mm}$ with a passive border of $8 \mathrm{~mm}$ on each side where the electrical connections (tin-coated conductive copper tape by $3 \mathrm{M}$ ) are attached. Overlapping fibers are removed with plastic tape before removing the mask. Uncovered areas are coated with micro glass spheres (by Suter Swiss Composite) to avoid having the borders sticking together when cutting the actuator from the frame. The membrane is then fixed to an insulated aluminum frame at the top and to a rectangular plate of PVC at the bottom. The necessary load to acquire the desired length is attached and the actuator is relaxed in that state for at least 12 hours. A voltage is applied with a ramp rate of $5 \mathrm{~V} / \mathrm{s}(\mathrm{HV}$-amplifier Trek $5 / 80)$ and the deformation is recorded with a video extensometer (LabView by National Instruments). The voltage is increased until electric breakdown occurs. The experimental data are compared with theoretical predictions (Fig. 7). The shear modulus used to fit is $45 \mathrm{kPa}$. The right-bottom inset shows configurations before and after applying voltage, corresponding to states (d) and (e) in Fig. 4. The left-top inset shows the conditions of loss of tension and electric breakdown. The loss of tension predicts a maximum actuation strain around 70\%, while in experiment a maximum actuation strain of $28 \%$ is obtained.

\subsection{Actuator made by sandwiching nylon fibers between two membranes}

Nylon fibers of diameter 0.2 mm (Clear Nylon line, Berkley Fishing) are placed parallel, with a gap of $2 \mathrm{~mm}$, on the top of a membrane of VHB 4905 (3M). Then, another layer of VHB 4905 is placed on the top and pushed down to stick to the previous one so that the nylon fibers are sandwiched between the two VHB membranes. The composite membrane consisted of the two VHB membranes and the fibers, is considered as a fiber-constrained membrane. Carbon grease is brushed on the top and bottom on the new membrane with 
active area $10 \mathrm{~cm} \times 4.1 \mathrm{~cm}$ and with a passive border of $5 \mathrm{~mm}$ on each side. Another fiber-constrained membrane is made in the same way, and then they are stuck together through the passive parts the edges in order to prohibit the air breakdown at the edges. The two electrodes facing each other and sealed between the fiber-constrained membranes are connected to the negative electrode and the other two electrodes were connected to the positive electrode of a high voltage source (Gamma High voltage Research, Model NO.: ES4OP-5W-DAM). The sample is stretched in the actuation direction under the dead loads for 30 minutes, and then a voltage was applied with a ramping rate of $50 \mathrm{~V} / \mathrm{s}$. A digital camera mounted horizontally is used to record the actuation of the sample until electric breakdown occurred. The experimental data are compared with theoretical predictions (Fig. 8). The shear modulus used to fit is $45 \mathrm{kPa}$. The right-bottom inset shows configurations before and after applying voltage, corresponding to states (d) and (e) in Fig. 4. The left-top inset shows the conditions of loss of tension and electric breakdown. The voltage needed in this experiment is much larger than that in the previous experiment. The experimentally measured voltage-stretch curves in both cases agree well those calculated theoretically. The maximum actuation strain is $17 \%$ predicted by the loss of tension, and is $25 \%$ as obtained in the experiment.

\subsection{Discussion on fiber-constrained dielectric elastomer actuators}

We note several differences between the theoretical assumptions and experimental behavior. First, the theory assumes a constant electric breakdown field independent of deformation, while experiments seem to show that electric breakdown field varies with deformation, ${ }^{16,29}$ although the cause of this dependence is still unclear.33 Second, the theory assumes that the membrane is elastic, but in experiment the VHB material shows significant viscoelasticity. 34,35 Third, the theory assumes that fibers fully constrain the horizontal deformation, in experiment this constraint is imperfect: fibers under compression can buckle, and fibers in tension implies that the membrane loses tension and possibly wrinkles. While the difference between the experimentally realized and theoretically predicted maximum actuation strains is unresolved at this writing, we note that the experimental voltage-strain curves agree well with those predicted theoretically, and that the 
experimentally achieved maximum strains are comparable to those of muscles, and should be adequate for many applications. A reviewer of this paper pointed out that fiber-stiffened dielectric elastomer membranes had been studied in a previous paper, and large uniaxial strains have also been demonstrated. ${ }^{36}$

\section{Conclusions}

The achievable voltage-induced deformation is strongly affected by how mechanical loads are applied. A theoretical comparison is made of actuators of three configurations. For a membrane under a uniaxial-force, the voltage-induced strain in the direction of applied force reduces as the force increases. For a membrane under equal-biaxial forces, the voltage-induced strain reaches a maximum at forces of an intermediate magnitude. For a membrane with stiffeners that restrict deformation in one direction, large voltage-induced deformation in the other direction is achievable. Experiments with membranes under equal-biaxial forces capture the theoretical predicted transition from failure limited by electromechanical instability to that limited by electric breakdown. Experiments with membranes under uniaxial forces capture the theoretical prediction that the maximum actuation decreases when the applied force increases. Experiments with fiber-constrained membranes give voltage-induced unidirectional strains up to $28 \%$. The experimentally observed voltage-stretch curves agree well with theoretical predictions. The experimentally achieved voltage-induced strains differ from those predicted theoretically, possibly due to imperfect constraint. A fiber-constrained membrane can be designed into any shape; in particular, the membrane can be made long in the direction of actuation, and narrow in the transverse direction. It is hoped that uniaxial constraint by means other than fibers will be explored, and that unidirectional actuation will soon be used in applications.

\section{Acknowledgements}

This work at Harvard is supported in Harvard by ARO (W911NF-09-1-0476), DARPA (W911NF-10-1-0113), and MRSEC. Lu is supported by China Scholarship Council as a visiting scholar for two years at Harvard University. The sabbatical of RH at Harvard was supported by the University of Texas at Austin through the Faculty Research Assignment 
program and by Institute of Computational Engineering and Science at the University of Texas through Moncrief Grand Challenge Award. 


\section{References}

1. $\quad$ R. Pelrine, R. Kornbluh, Q. B. Pei and J. Joseph, Science 2000, 287, 836-839.

2. F. Carpi, S. Bauer and D. De Rossi, Science 2010, 330, 1759-1761.

3. $\quad$ P. Brochu and Q. B. Pei, Macromol. Rapid. Commun. 2010, 31, 10-36.

4. I. A. Anderson, T. A. Gisby, T. McKay, B.M. O’Brien and E. Calius, J. Appl. Phys. In press (2012).

5. Q. B. Pei, R. Pelrine, S. Stanford, R. Kornbluh and M. Rosenthal, Synthetic Metals. 2003, 135, 129-131.

6. R. Shankar, T. K. Ghosh and R. J. Spontak, Adv. Mater. 2007, 19, 2218-2223.

7. G. Kovacs, L. Düring, S. Michel and G. Terrasi, Sens. Actuators A 2009, 155, 299-307.

8. S. Akbari and H.R. Shea, J. Micromech. Microeng. 2012, 22, 045020.

9. F. Carpi, G. Frediani, S. Turco and D. De Rossi, Adv. Func. Mater. 2011, 21, 4002-4008.

10. A. Cheng, $W W$-EAP Newsletter 2011, 13, 2.

11. T. Mckay, B. M. O'Brien, E. Calius and I. A. Anderson, Appl. Phys. Lett. 2010, 97, 062911.

12. R. Kaltseis, C. Keplinger, R. Baumgartner, M. Kaltenbrunner, T.F. Li, P. Mächler, R. Schwödiauer, Z. G. Suo and S. Bauer, Appl. Phys. Lett. 2011, 99, 162904.

13. J. S. Huang, T. F. Li, C. C. Foo, J. Zhu, D. R. Clarke and Z. G. Suo, Appl. Phys. Lett. 2012, 100, 041911.

14. C. Keplinger, T. F. Li, R. Baumgartner, Z. G. Suo and S. Bauer. Soft Matter 2012, 8, 285-288.

15. S. J. A. Koh, T. F. Li, J. X. Zhou, X. H. Zhao, W. Hong, J. Zhu and Z. G. Suo, J.Polym. Sci. Part B: Polym. Phys. 2011, 49, 504-515.

16. G. Kofod, R. Kornbluh, R. Pelrine and P. S. Larsen, J. Intel. Mater. Sys. Struct. 2003, 14, 787-793.

17. G. Kofod, J. Phys D: Appl. Phys. 2008, 41, 215405.

18. M. Kollosche, J. Zhu, Z. G. Suo and G. Kofod, Phys. Rev. E. Accepted for publication (2012).

19. M. Benslimane, H. E. Kiil and M. J. Tryson, Proc. SPIE 2010, 764231.

20. R. Kornbluh, pp 79-90 in Dielectric Elastomers as Electromechanical Transducers, 
edited by F. Carpi, D. D. Rossi, R. Kornbluh, R. Pelrine and P. S. Larsen. Elsevier, Oxford (2008).

21. D. Corbett and M. Warner, Sens Actuators A 2009, 149, 120-129.

22. R. Bustamante, Acta Mechanica 2009, 206, 237-259.

S. Rudykh, K. Bhattacharya and G. deBotton, Int. J. Non-Linear Mech. 2011, 62, $1131-1142$.

H. D. Yong, X. Z. He and Y. H. Zhou, Int. J. Eng. Sci. 2012, 50, 144-150.

25. 6o, 181-198.

28. A.N. Gent, Rubber. Chem. Tech. 1996, 69, 59-61.

29.

30. 20, 075003 013517. Electroactive Polymer Actuators and Devices (EAPAD). Edited by Bar-Cohen, Yoseph. Proceedings of the SPIE, Volume 6168, pp. 27-38 (2006). DOI: 10.1117/12.677981. 


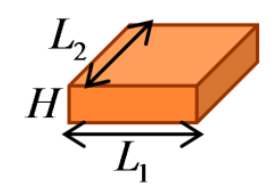

Reference state

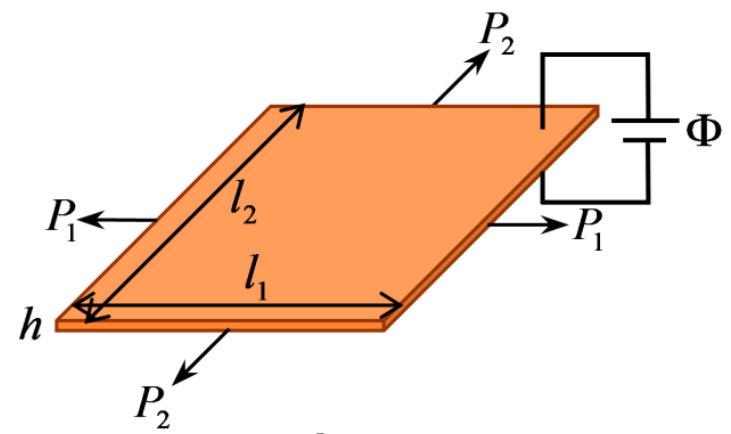

Actuated state

Fig. 1 Schematics of a membrane of a dielectric elastomer. In the reference state, subject to no force and no voltage, the membrane is of thickness $H$ and lengths $L_{1}$ and $L_{2}$. In the actuated state, subject to forces $P_{1}$ and $P_{2}$ in the plane and voltage $\Phi$ through the thickness, the membrane deforms to thickness $h$ and lengths $l_{1}$ and $l_{2}$. 


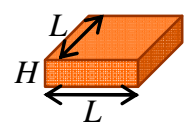

Reference state

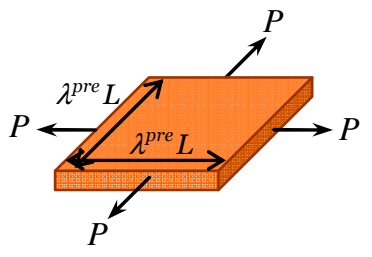

Prestretched state

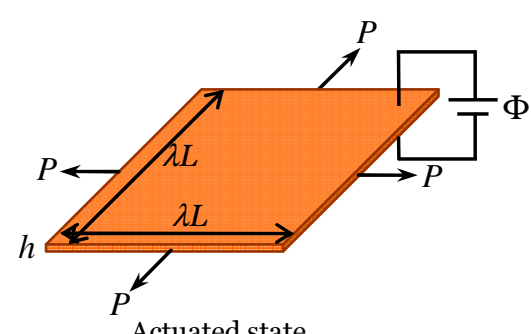

Actuated state

(a)

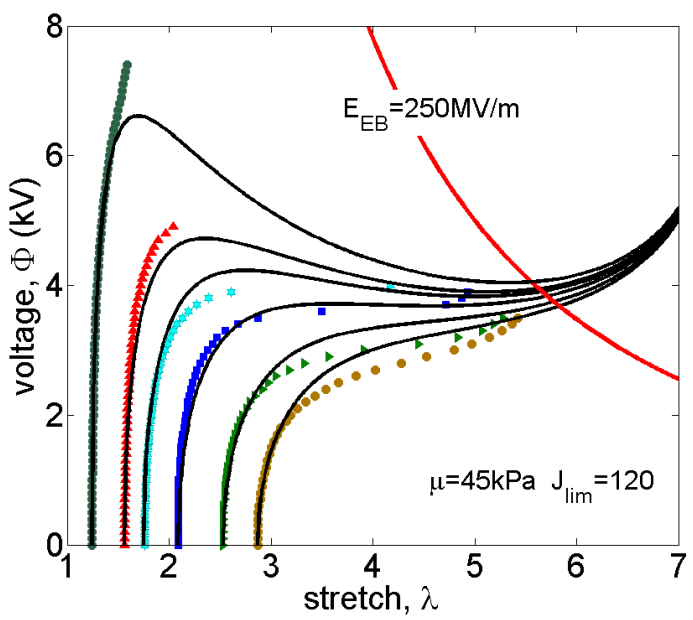

(b)

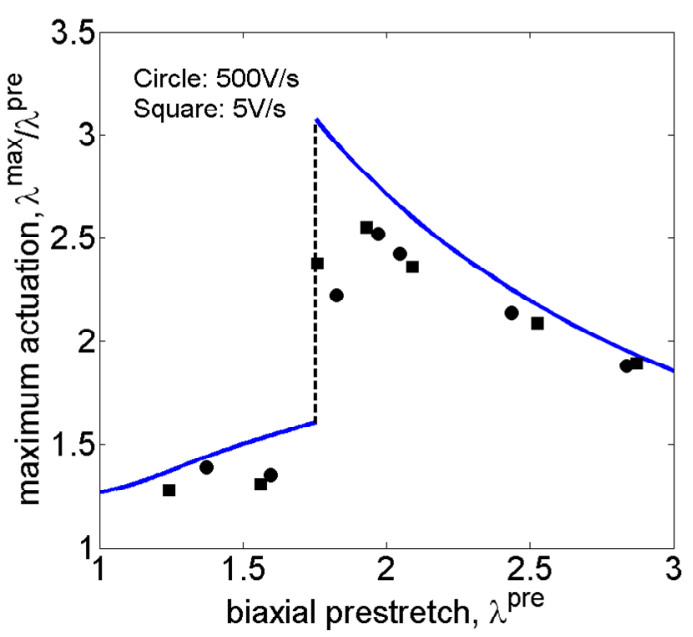

(c)

Fig. 2 Actuation of a membrane under the equal-biaxial forces. (a) Several states are illustrated for a membrane under the equal-biaxial conditions. (b) For a given prestretch $\lambda^{\text {pre }}$, as the voltage $\Phi$ varies, the membrane changes in a succession of states of equilibrium, represented by a curve in the voltage-stretch diagram. The value of $\lambda^{\text {pre }}$ for each curve can be read from the intersection between the curve and the horizontal axis. The theoretical curves are compared with the experimental data. Also included is the curve representing the conditions of electric breakdown. (c) The maximum actuation $\lambda^{\max } / \lambda^{\text {pre }}$ is plotted as a function of the prestretch $\lambda^{\text {pre }}$. Actuation is limited by electromechanical instability for small $\lambda^{\text {pre }}$, and is limited by electric breakdown for large $\lambda^{\text {pre }}$. The theoretical predictions are compared with the experimental data. 


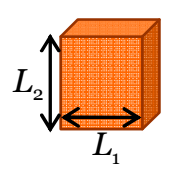

Reference state

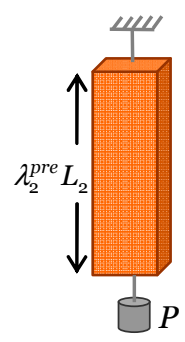

Prestretched state

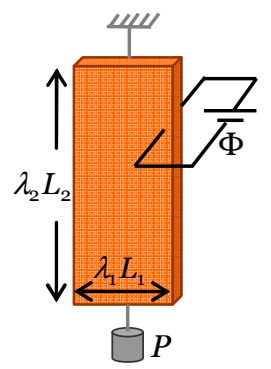

Actuated state

(a)

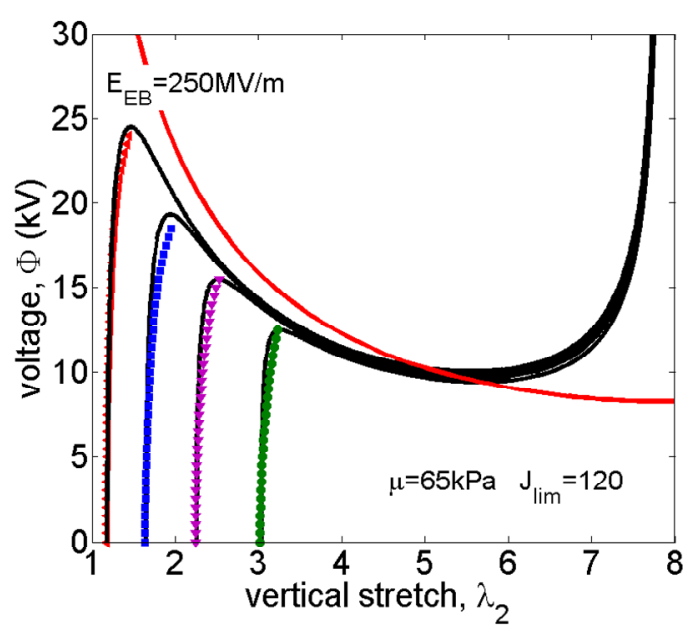

(b)

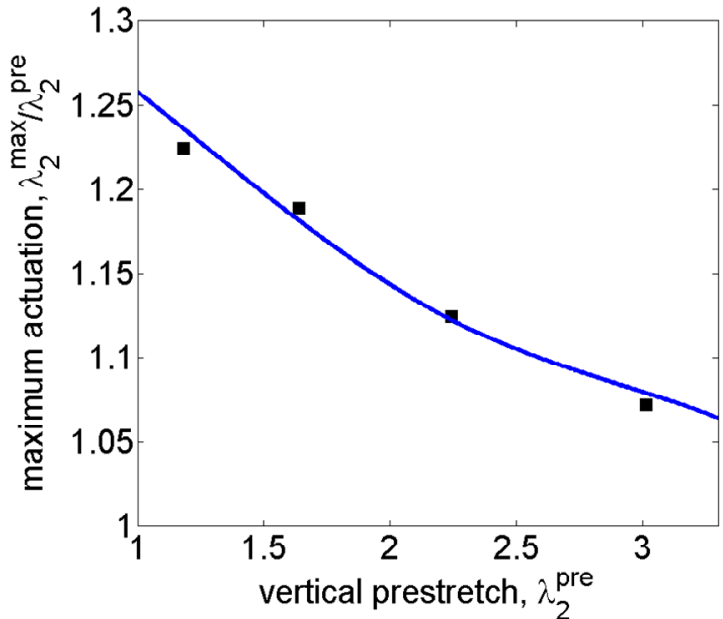

(c)

Fig. 3 Actuation of a membrane under a uniaxial force. (a) Several states are illustrated for a membrane under a uniaxial force. (b) For a given prestretch $\lambda_{2}^{\text {pre }}$, as the voltage $\Phi$ varies, the membrane changes in a succession of states of equilibrium, represented by a curve in the voltage-stretch diagram. The value of $\lambda_{2}^{\text {pre }}$ for each curve can be read from the intersection between the curve and the horizontal axis. The theoretical curves are compared with experimental data. Also included is the curve representing the conditions of electric breakdown. In all cases plotted, the maximum actuation is limited by electromechanical instability. (c) The maximum actuation $\lambda_{2}^{\max } / \lambda_{2}^{\text {pre }}$ is plotted as a function of the prestretch $\lambda_{2}^{\text {pre }}$. The theoretical predictions are compared with the experimental data. 


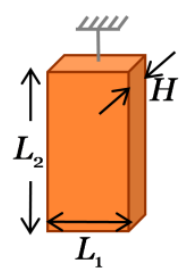

(a)

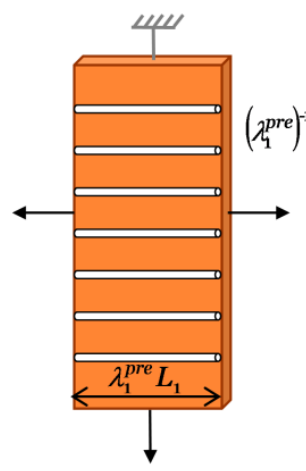

(b)
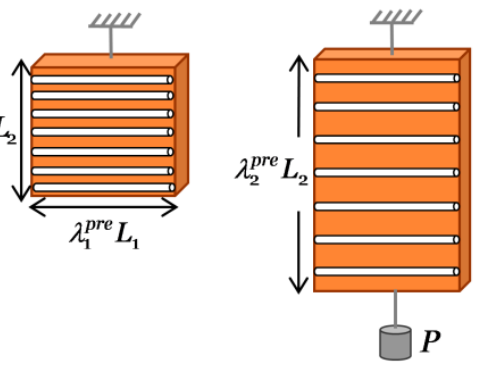

$(d)$

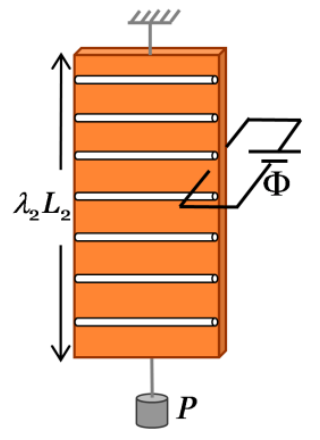

(e)

Fig. 4 An actuator made of a dielectric membrane stiffened with fibers. (a) In the reference state, the membrane is not stretched. (b) The membrane is pre-stretched in both directions and fibers are added. (c) When the mechanical forces are released, the membrane contracts in the directions normal to the fibers, but keep dimension in the direction along the fibers. (d) The membrane is subject to a force $P$. (e) The membrane is subject to both the force and voltage $\Phi$. 

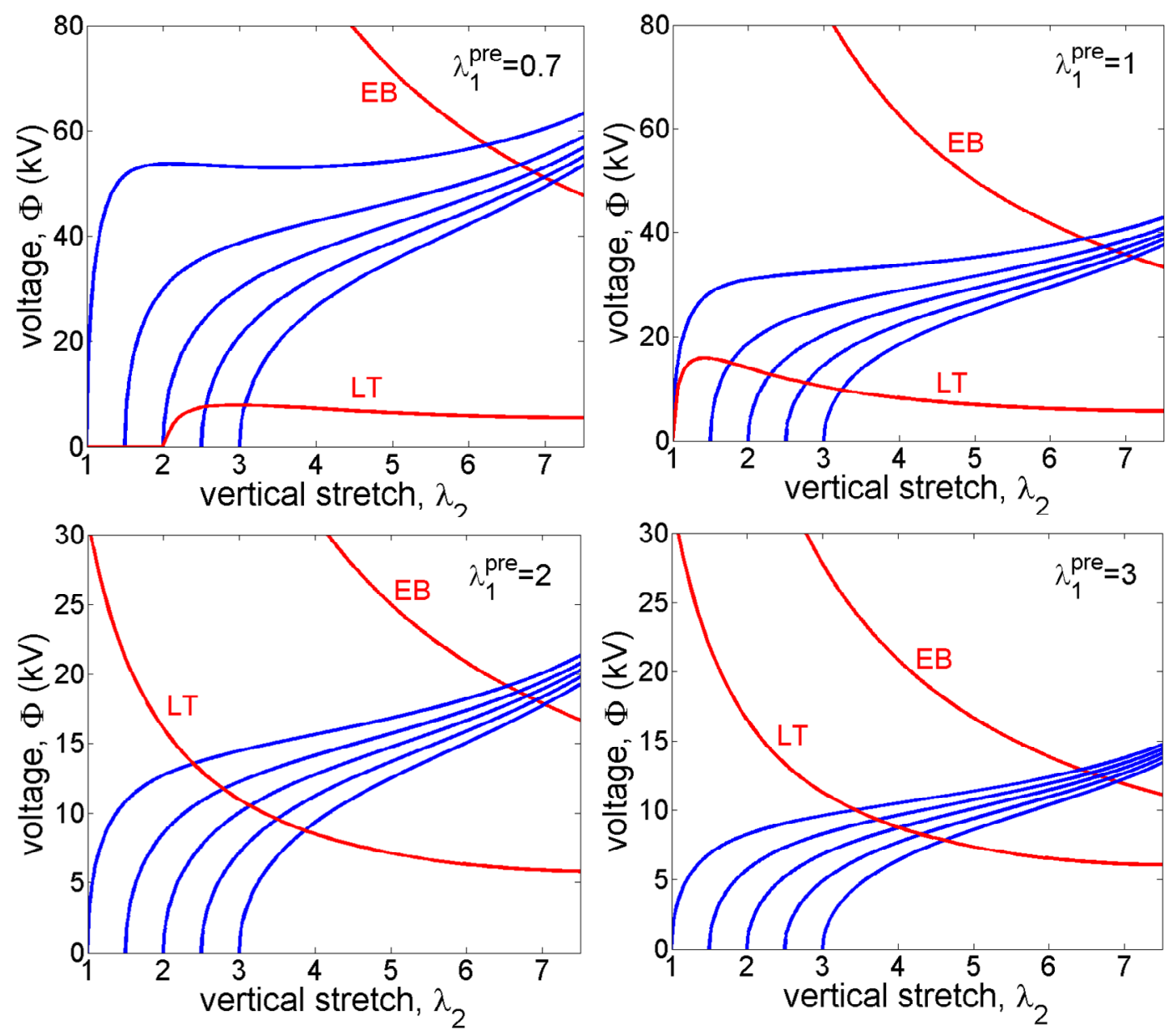

Fig. 5 Actuation of a fiber-constrained membrane of several values of the prestretch. Also marked are conditions of electric breakdown (EB) and conditions of loss of tension (LT). 


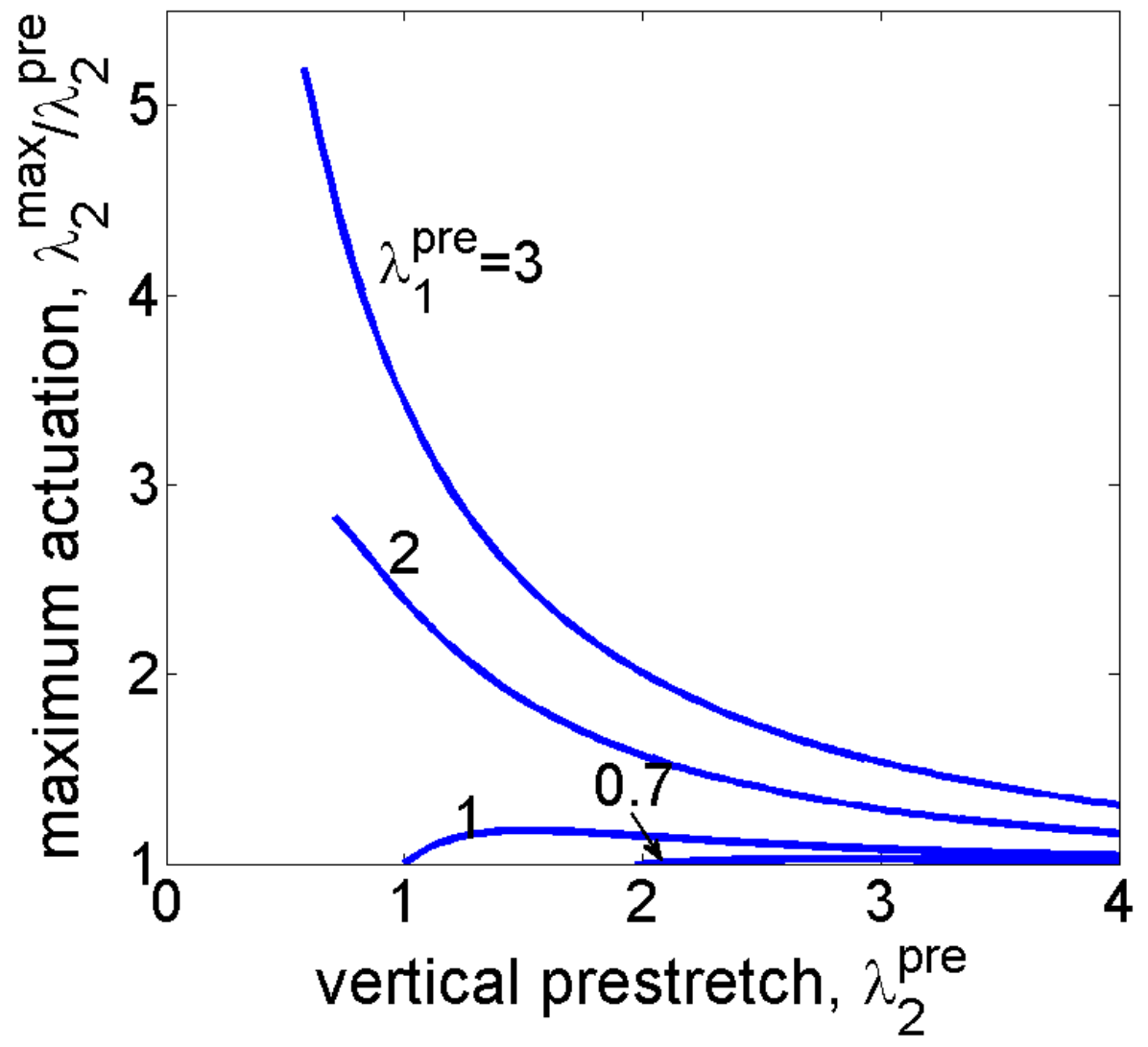

Fig. 6 Maximum actuation of a fiber-constrained membrane. The maximum actuation $\lambda_{2}^{\max } / \lambda_{2}^{\text {pre }}$ is plotted as a function of the vertical prestretch $\lambda_{2}^{\text {pre }}$ for several values of the horizontal prestretch $\lambda_{1}^{\text {pre }}$. 


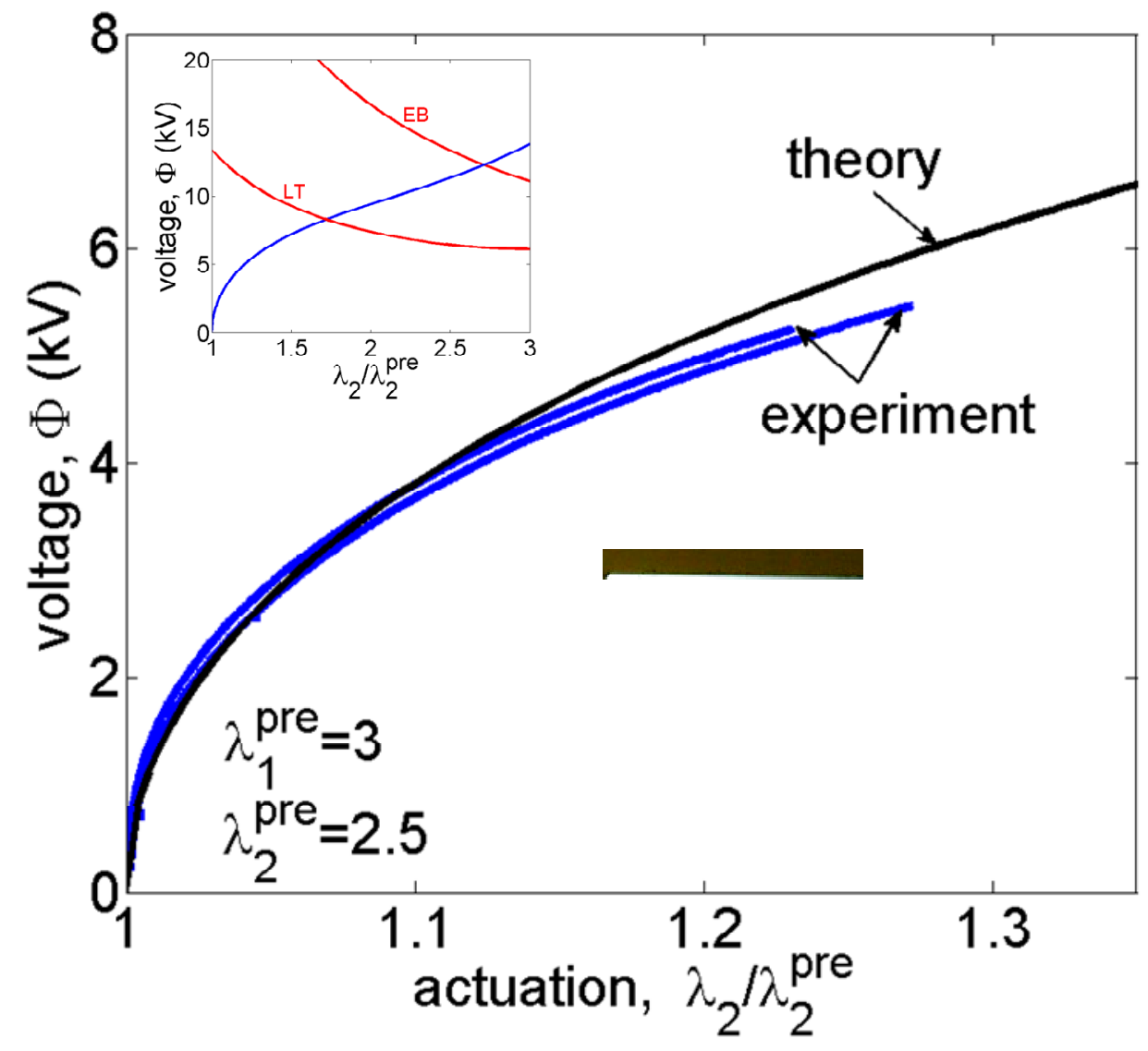




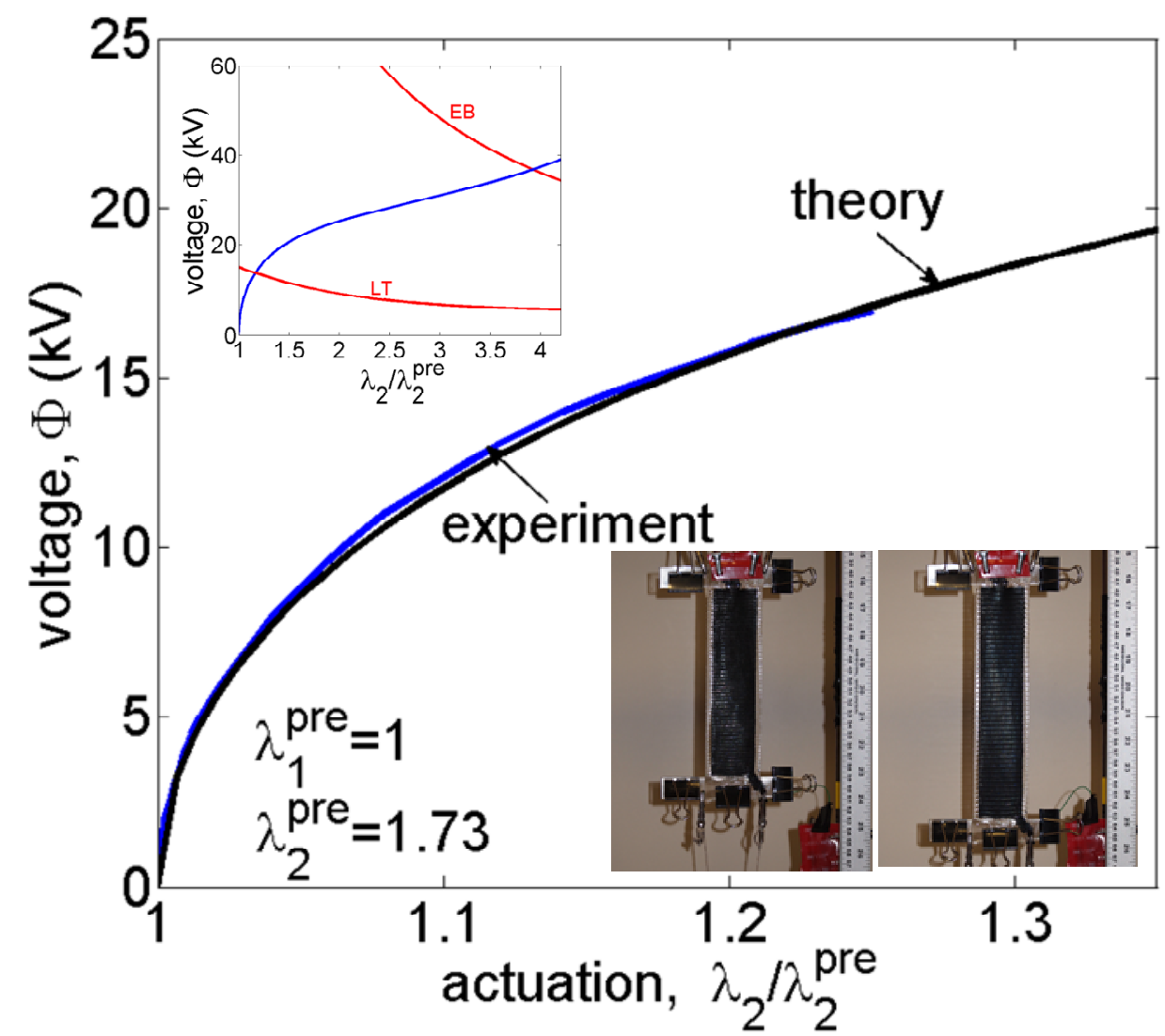

Fig. 8 Actuator made by sandwiching nylon fibers between two membranes. Also shown are the conditions of loss of tension (LT) and electric breakdown (EB), as well as photographs of the experiment. 